\title{
Spatially Resolved Deformation Studies on Carbon Steel employing X-rays and Positron Annihilation
}

\author{
Matz Haaks ${ }^{*}, 1$, Ingo Müller ${ }^{1}$, Andreas Schöps ${ }^{2}$, Hermann Franz ${ }^{2}$ \\ ${ }^{1}$ Helmholtz Institut für Strahlen- und Kernphysik, Nußallee 14-16, 53115 Bonn, Germany \\ ${ }^{2}$ DESY/HASYLAB, Notkestr. 85, 22603 Hamburg, Germany
}

PACS: 61.72.Dd, 81.40.Lm, 78.70.Bj, 07.78.+s, 61.10.Nz, 75.50.Bb

*Corresponding author: e-mail haaks@iskp.uni-bonn.de

\begin{abstract}
Spatially resolved studies on plasticity in a polycrystalline sample of the ferritic carbon steel C45E (AISI 1045) were performed after deformation in a three-point bending test. The local effects of deformation were investigated by scanning the sample with two different methods: Positron Annihilation Spectroscopy (S-parameter) and De-
\end{abstract}

bye-Scherrer diffraction (reflex broadening). A simple relation between the results of both experiments and the true strain over the cross-section of the bent sample is presented in this letter. Comparing the methods a linear correlation between the lattice distortion of $\alpha$-iron and the defect sensitive positron annihilation parameter is found.
1 Introduction In most cases plastically deformed metals show a strongly inhomogeneous defect distribution in the deformed region. Hence, to understand the physics behind, spatially resolved information of the material properties like defect concentration, or internal stress and strain are required. In this letter we present two experimental methods both scanning over the deformed region of exactly the same sample.

As a simple example for elastic-plastic deformation, a three-point bending test was performed in the commonly used ferritic steel C45E (equal to AISI 1045). This geometry is characterised by a linear stress gradient along the bending radius and a neutral fibre between elongation and contraction. The remaining plastic deformation after releasing the stress was measured with Positron Annihilation Spectroscopy (PAS) employing the $S$-parameter derived from Doppler Broadening of the Annihilation Radiation (DBAR), which is highly sensitive on the defect density in deformed metals. Generally, DBAR measurements provide information about the momentum of the annihilating electron-positron pair. Trapped in a vacancy or a vacancy-like defect, the positron sees a different momentum distribution than when annihilating in the interstitial region (free positron state) [1-3]. Plastic deformation is always accompanied by the production of associated, non-equilibrium point defects like vacancies. Hence, the dislocation density is accessed via the change in the Doppler broadening caused by trapping into the associated vacancies [4].

The internal stress was measured in a Debye-Scherrer experiment at the hard X-ray beam-line at PETRA II at the DESY/HASYLAB [5] in a similar setup as reported in [6]. In contrast to the authors we performed spatially resolved $\mathrm{X}$-ray measurements using a tiny beam spot. Employing $\mathrm{X}$-ray diffraction, the information on the internal stresses and strains originating from the constituents of an alloy, in our case $\alpha$-iron and $\mathrm{Fe}_{3} \mathrm{C}$, can be separated.

2 Experimental A flat-bar sample with a crosssection of $3 \times 10 \mathrm{~mm}^{2}$ was cut from a block of C45E and subsequently mechanically polished down to a residual roughness of $\sim 1 \mu \mathrm{m}$. To receive a reproducible initial state for the further deformation, the sample was subjected to a heat treatment for $3 \mathrm{~h}$ at $680^{\circ} \mathrm{C}$ in a $2 \times 10^{-6}$ mbar vacuum (cooling rate: $1 \mathrm{~K} / \mathrm{min}$ ). After this treatment the material was in a fine-grained state consisting of $60 \%$ perlite and $40 \% \alpha$-iron, with an average grain size of $20 \mu \mathrm{m}$. In this well-annealed state the defect concentration is below the sensitivity threshold for positrons [7]. The sample was bent over the larger axis to a resulting radius of $55 \mathrm{~mm}$, using a hydraulic press with cylindrically shaped plungers. 
The DBAR measurements were performed at the Bonn Positron Microprobe (BPM) [8] with a positron energy of $30 \mathrm{keV}$ collecting $3 \times 10^{5}$ events in the $511 \mathrm{keV}$ annihilation peak. The beam diameter was adjusted to $100 \mu \mathrm{m}$ to average over several grains. The spectra were taken in a cross-sectional scan along the bending radius in steps of $100 \mu \mathrm{m}$. Stabilisation of the electronics and spectral background subtraction was done according to the methods described in [9]. The $S$-parameter was evaluated in a energy range of $\pm 1.38 \mathrm{keV}$ around $511 \mathrm{keV}$.

The Debye-Scherrer experiment was performed with a X-ray energy of $67.2 \mathrm{keV}$. The beam profile was adjusted to $1.5 \times 0.1 \mathrm{~mm}^{2}$ with the larger dimension in direction perpendicular to the stress gradient in the sample. In this volume the stress can be assumed to be uniform. Hence, the effect of the bending deformation is measured with a resolution of $100 \mu \mathrm{m}$ in radial direction in an irradiated volume of $0.45 \mathrm{~mm}^{3}$, containing $\sim 4 \times 10^{4}$ grains. The Debye-rings were recorded by an image plate detector (Mar345, Mar Instruments, Evanston, IL, USA) with a resolution of $2300 \times 2300$ pixels with 16 bit information depth. With a sample to detector distance of $1000 \mathrm{~mm}$ diffraction angles up to $2 \Theta=9.6^{\circ}$ could be recorded. This area includes the first three reflections of $\alpha$-iron and several reflections of $\mathrm{Fe}_{3} \mathrm{C}$. The samples diameter was scanned in steps of $100 \mu \mathrm{m}$ with an exposure time of $10 \mathrm{~s}$. In the beginning of the series reference spectra of $\mathrm{LaB}_{6}$ were taken for calibration. The two-dimensional data were first integrated azimuthally from $75^{\circ}$ to $105^{\circ}\left(90^{\circ}\right.$ equals to the bending direction) using the software Fit2d [10]. In the resulting onb-dimensional spectra of the diffraction angle $2 \Theta$ a smooth background caused by the sample holder (PMMA) was fitted and subtracted. The diffraction angle was converted into lattice spacing using the Braggequation. Amplitudes, positions and line widths (FWHM) of the reflections were fitted applying a Gaussian peak model.

3 Results The DBAR scan over the bending radius is shown in Fig. 1. The $S$-parameter is displayed normalised to the $S$-parameter measured in the annealed state of the material. In the neutral fibre, which is located $-0.1 \mathrm{~mm}$ next to the centre of the sample, the $S$-parameter does not differ significantly from the reference value measured in the annealed state, which yields in the absence of plastic deformation here. From the centre, the $S$-parameter rises towards the edges of the sample in both directions, showing only a slight asymmetry. The $S$-parameter is sensitive to all irreversible changes in the open volume of the lattice. Hence, in the whole sample, despite of the neutral fibre, the deformation is plastic. In a first assumption of threepoint bending, the engineering bending strain $\varepsilon_{\text {eng }}$ along the bending radius can be assumed to vary linearly along the cross-section of the sample. The true bending strain $\mathcal{E}_{\text {true }}$ can be calculated according to a simple formula [11]:

$$
\varepsilon_{\text {true }}=\ln \left(1+\varepsilon_{\text {eng }}\right)
$$

In-situ measurements of the $S$-parameter in tensile tests were performed on annealed C45E and reported in [7].

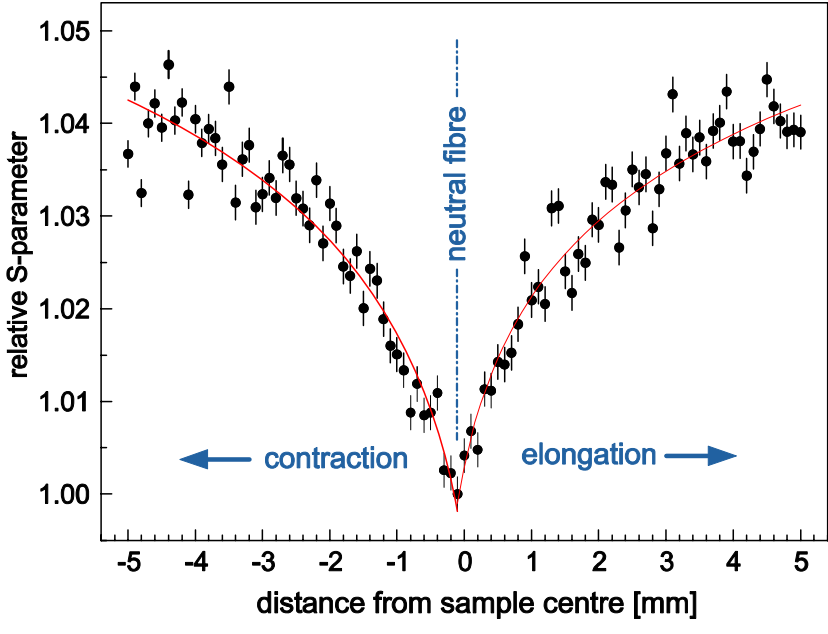

Figure 1 Spatially resolved scan of the relative $S$-parameter over the cross-section of the bent sample (full circles) together with a fit considering a linear dependency of the $S$-parameter to the true strain (full lines). The origin of the abscissa is set in the geometrical centre of the sample.

In the plastic region of the stress-strain diagram, beyond the Lüders region, the $S$-parameter shows a linear relation on the true strain.

The fit in fig. 1 (full lines) were performed using this simple model (1) following the formula for the true strain:

$$
S=1+p_{1} \ln \left(1+p_{2} x\right)
$$

Here $S$ is the relative $S$-parameter, $p_{1}, p_{2}$ are fitting parameters and $x$ is the distance from the neutral fibre.

The lattice distortion of three crystallographic planes of $\alpha$-iron, corresponding to the first three reflections are shown in Fig. 2. Generally, the shapes of the curves for the $\{200\}$ and $\{211\}$ planes follow the shapes of the $S$-parameter (Fig.1).

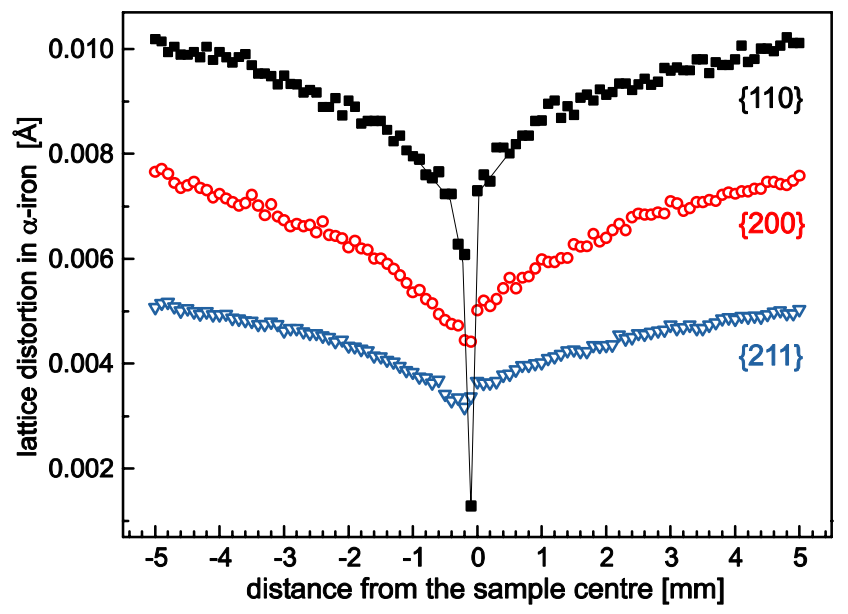

Figure 2 Lattice distortion in the $\alpha$-phase of C45E: Broadening (FWHM) of the lattice constant distribution of three crystallographic planes. The scan is performed at the same positions as the DBAR measurements. The error bars are of the same size as the symbols. 


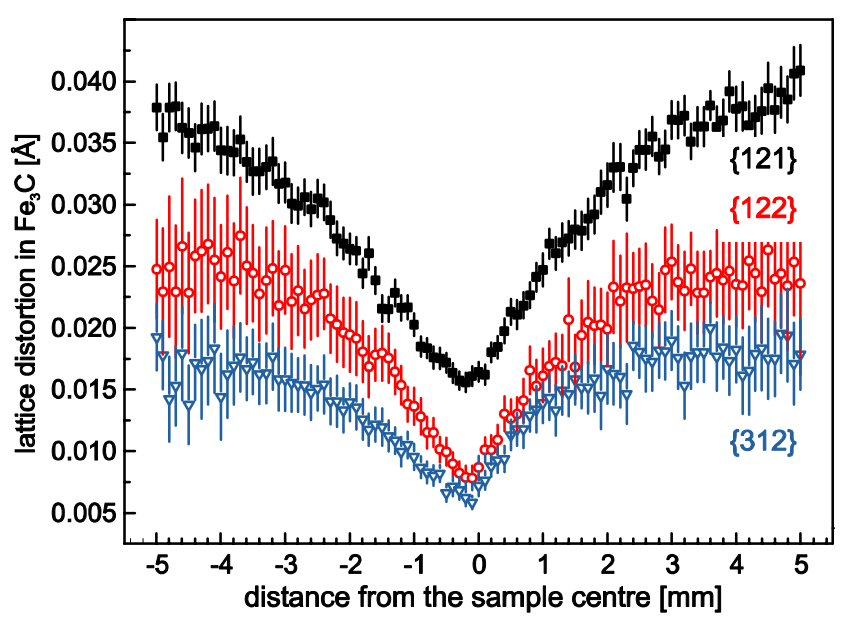

Figure 3 Lattice distortion in $\mathrm{Fe}_{3} \mathrm{C}$ : Broadening (FWHM) of the lattice constant distribution of three crystallographic planes.

The $\{110\}$ planes are the simplest slip planes in $\alpha$-iron and hence are activated first in simple glide causing the sharp notch in the plot. Close to the neutral fibre this causes no distortion on these planes. Since the $\{200\}$ planes are no slip system, their broadening is due to the strain caused by dislocations in the other slip systems and climbing of dislocations.

Figure 3 shows the lattice distortion for $\mathrm{Fe}_{3} \mathrm{C}$ from the perlitic phase. The effect is three times larger compared to the $\alpha$-iron data, since the strain in the $\mathrm{Fe}_{3} \mathrm{C}$ phase is not reduced by dislocations The curves show a smoother shape in the central region, which indicates the absence of simple slip.

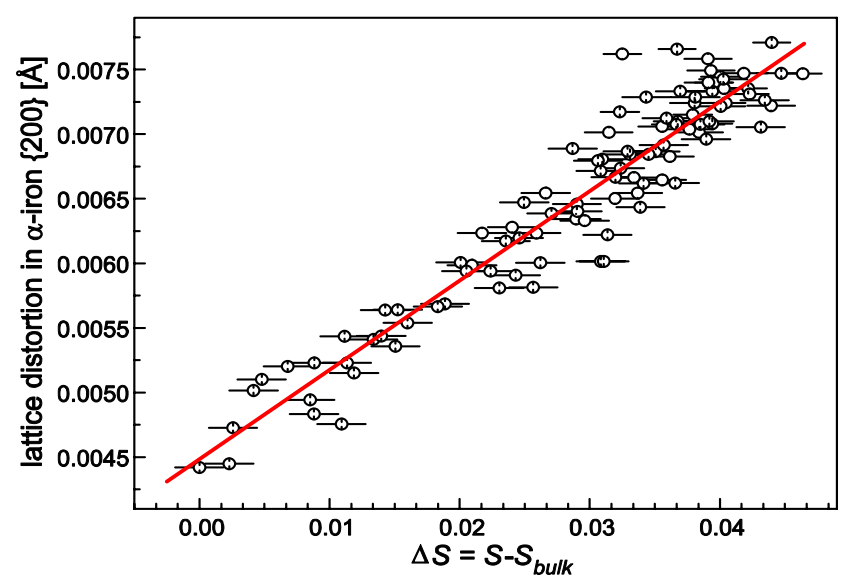

Figure 4 Lattice distortion of the $\{200\}$ planes of $\alpha$-iron vs. the reduced S-parameter (circles) and linear fit (solid line).
4 Conclusion As a simple example for plastic deformation in metals and alloys, this three-point bending test has shown the advantage of spatially resolved studies of either the defect density and the lattice distortion.

Due to the high energy available and the high brilliance of the PETRA II beam-line, Debye-Scherrer diffraction was possible even on a $3 \mathrm{~mm}$ thick polycrystalline metal sample. A spatial resolution of $100 \mu \mathrm{m}$ could be achieved, having still a sufficient number of grains in the irradiated volume for accurate data evaluation.

A comparison of the Debye-Scherrer data and the DBAR data shows the same dependence for both methods on the position and hence on the true strain in the bent sample, which can be estimated according to the simple formula (1).

The broadening of the reflections is connected with the lattice distortion caused by the movement and multiplication of dislocations, while the $S$-parameter depends on the dislocation density. Hence, both experimentally accessible values are based upon on the same physical phenomenon. This can be expressed in a linear correlation, as displayed in Fig. 4 exemplarily for the distortion of the $\{200\}$ planes of the $\alpha$-phase vs. the reduced S-parameter $\Delta S=S-S_{\text {bulk }}$.

Acknowledgements We would like to thank Stephan V. Roth and Karel Saksl for their help before and during the experiment at the DESY/HASYLAB.

\section{References}

[1] A. Seeger, J. Phys. F 3, 248 (1973).

[2] L. C. Smedskjær and M. J. Fluss, in: Methods of Experimental Physics, Vol. 21, edited by R. Celotta and J. Levine (Academic Press, Orlando, 1983), chap. 2, p. 77.

[3] K. G. Lynn, J. R. McDonald, R. A. Boie, L. C. Feldman, J. D. Gabbe, M. F. Robbins, E. Bonderup, and J. Golovchenko, Phys. Rev. Lett. 38, 241 (1977).

[4] G. Saada, Acta Metall. 9, 965 (1961).

[5] K. Balewski, W. Brefeld, U. Hahn, J. Pflüger, and R. Rosmanith, Proc. Particle Accel. Conf. 1, 275 (1995).

[6] J. Böhm, A. Wanner, R. Kampmann, H. Franz, K. D. Liss, A. Schreyer, and H. Clemens, Nucl. Instrum. Methods B 200, 315 (2003).

[7] K. Bennewitz, M. Haaks, T. Staab, S. Eisenberg, Th. Lampe, and K. Maier, Z. Met.kd. 93, 778 (2002).

[8] H. Greif, M. Haaks, U. Holzwarth, U. Männig, M. Tongbhoyai, T. Wider, K. Maier, H. Bihr, and B. Huber, Appl. Phys. Lett. 15, 2115 (1997).

[9] M. Haaks, T. E. M. Staab, K. Saarinen, and K. Maier, phys. stat. sol. (a) 202, R38 (2005).

[10] A. P. Hammersley, ESRF Internal Report, ESRF97HA02T, Fit2d: An Introduction and Overview (1997).

[11] P. Dadras, in: ASM Handbook, Vol. 8: Mechanical Testing and Evaluation, edited by H. Kuhn et al. (ASM International, Material Park, OH, 2000), p. 109. 